\title{
A Wideband Differentially-Fed Dual-Polarized Antenna with Stable Radiation Pattern for Base Stations
}

\author{
Ding-Liang Wen, Dong-Ze Zheng and Qing-Xin Chu, Senior Member, IEEE
}

\begin{abstract}
A new wideband differentially-fed dual-polarized antenna with stable radiation pattern for base stations is proposed and studied. A cross-shaped feeding structure is specially designed to fit the differentially-fed scheme and four parasitic loop elements are employed to achieve a wide impedance bandwidth. A stable antenna gain and a stable radiation pattern are realized by using a rectangular cavity-shaped reflector instead of a planar one. A detailed parametric study was performed to optimize the antenna's performances. After that, a prototype was fabricated and tested. Measured results show that the antenna achieves a wide impedance bandwidth of $52 \%$ with differential SWR $<1.5$ from 1.7 to $2.9 \mathrm{GHz}$ and a high differential port-to-port isolation of better than $26.3 \mathrm{~dB}$ within the operating frequency bandwidth. A stable antenna gain $(\approx 8 \mathrm{dBi})$ and a stable radiation pattern with 3-dB beamwidth of $65^{\circ} \pm 5^{\circ}$ were also found over the operating frequencies. Moreover, the proposed antenna can be easily built by using PCB fabrication technique due to its compact and planar structure.
\end{abstract}

Index Terms-Differentially-fed antenna, wideband antenna, base station antenna, dual-polarization, stable radiation pattern.

\section{INTRODUCTION}

$\mathrm{R}$ APID development of modern communications makes it desirable to have antennas satisfying some stringent requirements, such as wide impedance bandwidth, unidirectional radiation, stable gain, stable radiation pattern, low profile, etc. For cellular wireless communication systems, $\pm 45^{\circ}$ dual-polarized base station antennas are widely applied especially in large cities, where a huge communication channel capacity and a high data transmission speed are required. Recently, large numbers of dual-polarized base station antennas have been proposed [1-6]. Unfortunately, these antennas still suffer from a narrow impedance bandwidth. In 2014, a wideband dual-polarized base station antenna was investigated [7]. Good impedance matching, good port-to-port isolation, stable radiation pattern, and stable gain are obtained for the antenna [7] from 1.7 to $2.7 \mathrm{GHz}$. Other dual-polarized

Manuscript received February \#\#, 2016. This work was supported by the National Natural Science Foundation of China (61171029), the Guangzhou Science and Information Technology Project (201300000029) and the Fundamental Research Funds for the Central Universities (2013ZP0018).

The authors are with the School of Electronic and Information Engineering, South China University of Technology, Guangzhou 510640, China Correspondence and requests for materials should be addressed to Q. X. Chu (e-mail: qxchu@scut.edu.cn). base station antennas operating between 1.7 and $2.7 \mathrm{GHz}$ have also been developed in the recent five years [8-10].

Increasing demand in the wireless communication market has led to the need for compact and fully integrated radio frequency (RF) front-end products, for which differential signals are more preferable. However, most of current antennas, such as antennas proposed in [2-10], are designed to be single-ended, which means that a balun or a $180^{\circ}$ hybrid is usually needed to connect them to the differential microwave circuits. Unfortunately, adding a balun or a $180^{\circ}$ hybrid will cause additional losses, increase the whole circuit's size and reduce the antennas' impedance bandwidth. To avoid all of these weaknesses, differentially-fed antennas are required, with which a balun or a $180^{\circ}$ hybrid is no longer necessary.

Recently, a large number of papers about differentially-fed antennas have been presented [11-16]. However, most of them [11-14] are single-polarized. Although both of the antennas proposed in $[15,16]$ are dual-polarized, the former one has a narrow impedance bandwidth while the latter one's radiation pattern is not unidirectional. In 2013, a differentially-fed magneto-electric dipole antenna was proposed [17], which achieves a wide impedance bandwidth of $114 \%$. Stable unidirectional radiation patterns are also achieved over the most of the operating frequency band. In the same year, a novel differentially-driven dual-polarized magneto-electric dipole antenna was also developed by Prof. Q. Xue and his team [18]. A wide impedance bandwidth of $68 \%$ for differential reflection coefficients less than $-10 \mathrm{~dB}$ and stable radiation patterns are obtained across the operating frequency band.

This paper proposes a new wideband differentially-fed dual-polarized antenna with a theoretically infinite differential port-to-port isolation. A cross-shaped feeding structure [18] is specially designed to fit the differentially-fed scheme. Four parasitic loop elements are added to shift the upper resonance of the antenna to a lower frequency. As a result, the antenna can achieve a wide impedance bandwidth from 1.7 to $2.9 \mathrm{GHz}$ with differential $\mathrm{SWR}<1.5$. Great differential port-to-port isolation, stable gain and stable radiation pattern are also realized within the operating frequency band. 


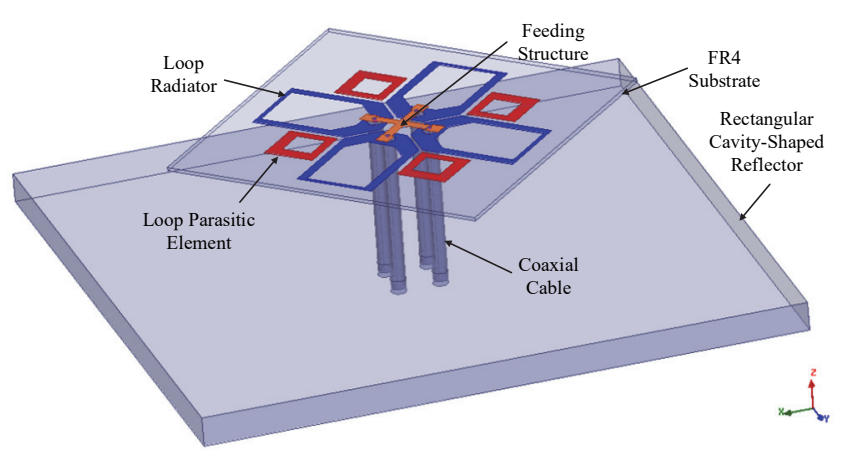

(a) 3D view

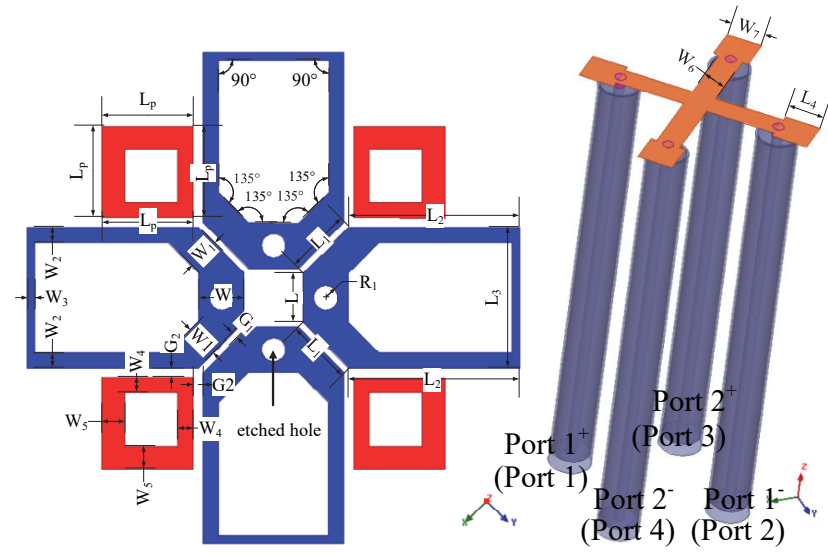

(b) perspective view

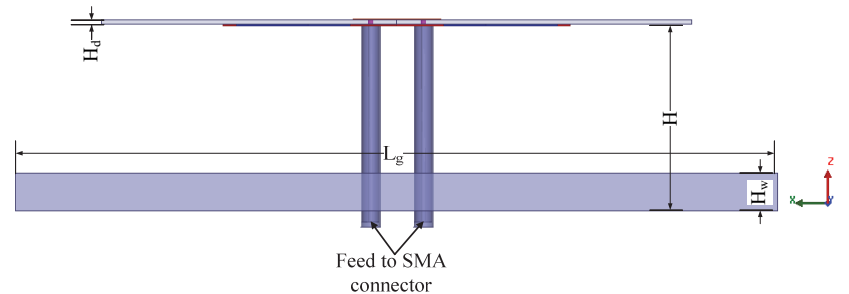

(c) side view

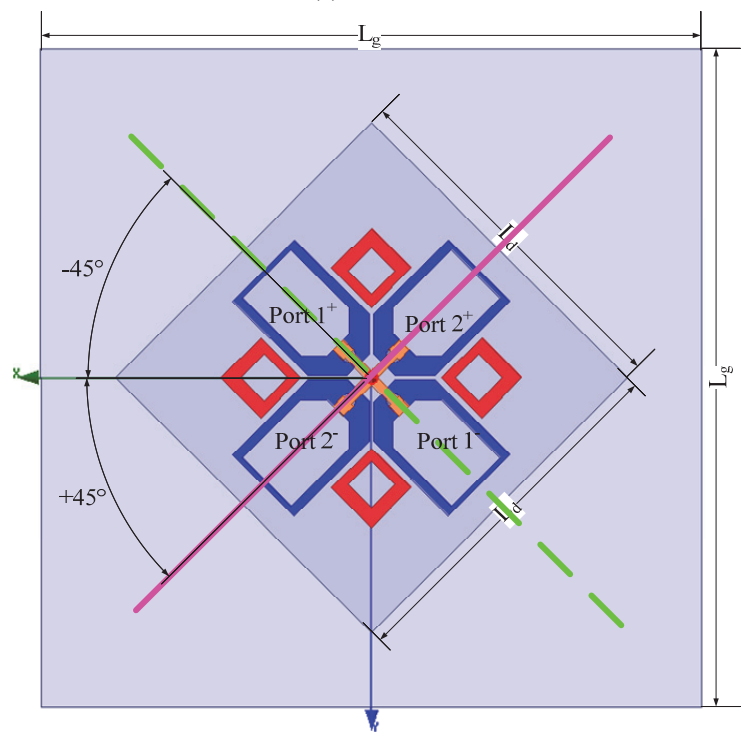

(d) top view

Fig. 1. Configuration of the proposed differentially-fed dual-polarized antenna: $\mathrm{L}_{\mathrm{g}}=140, \mathrm{~L}_{\mathrm{d}}=76, \mathrm{~L}=6.5, \mathrm{~L}_{1}=8.5, \mathrm{~L}_{2}=22.5, \mathrm{~L}_{3}=18.52, \mathrm{~L}_{4}=3, \mathrm{~L}_{\mathrm{p}}=12, \mathrm{H}=34, \mathrm{H}_{\mathrm{d}}=0.8$, $\mathrm{H}_{\mathrm{w}}=6, \mathrm{~W}=6, \mathrm{~W}_{1}=4.24, \mathrm{~W}_{2}=2, \mathrm{~W}_{3}=1, \mathrm{~W}_{4}=2, \mathrm{~W}_{5}=3, \mathrm{~W}_{6}=2, \mathrm{~W}_{7}=3, \mathrm{G}_{1}=0.85$, $\mathrm{G}_{2}=1.3, \mathrm{R}_{1}=1.58$ (Unit: $\mathrm{mm}$ ).

\section{ANTENNA DESIGN}

Fig. 1 shows the geometry of the proposed differentially-fed dual-polarized antenna. After a detailed parametric study, the optimized dimensions of the antenna are acquired, which are given in the final annotation of Fig. 1. The parametric study will be presented in Section III. In order to correspond to the position of base station antenna for real applications, xoz-plane and yoz-plane are defined as the horizontal and vertical plane, respectively. As can be seen from the $3 \mathrm{D}$ view of the antenna, depicted in Fig. 1(a), the antenna is composed of two pairs of orthogonal hexagonal loop radiators, four quadrangular loop parasitic elements, a cross-shaped feeding structure, a rectangular cavity-shaped reflector and four coaxial feeds. Fig. 1(a) also indicates that the antenna is $90^{\circ}$ rotationally symmetrical. The loop radiators, the loop parasitic elements and the feeding structure are built on the FR4 substrate with a $\varepsilon_{\mathrm{r}}$ $=4.4$, a $\tan \delta=0.02$, and a thickness $=0.8 \mathrm{~mm}$. The loop radiators and the loop parasitic elements are printed on the lower side of the substrate while the feeding structure is on the upper side.

As illustrated in Fig. 1(b), four coaxial feeds are employed to excite the antenna. The cross-shaped feeding structure is connected to the inner conductors of the four $50-\Omega$ coaxial cables through the substrate. On the other hand, the outer conductors of the coaxial cables are soldered on the loop radiators. Fig. 1(b) also shows that an etched hole is made on each loop radiator to avoid intersection.

The side view of the antenna is depicted in Fig. 1(c). A SMA connector is bonded to each of the coaxial cable under the ground plane. Therefore, four SMA connectors are needed and these four ports are defined as single-ended port 1, 2, 3 and 4, respectively, as illustrated in Fig. 1(b). Accordingly, single-ended port 1 and port 2 are designated as differential port 1 while single-ended port 3 and 4 are designated as differential port 2 . To excite a differential port, the signals with the same amplitude and $180^{\circ}$ phase difference should be transmitted to the two corresponding ports, such as port $1^{+}$and port $1^{-}$, or port $2^{+}$and port $2^{-}$. As shown in Fig. 1(d), when differential port 1 is excited, the antenna is $-45^{\circ}$ polarized along the dashed line direction; when differential port 2 is excited, the antenna is $+45^{\circ}$ polarized along the solid line direction.

The principle of this antenna's wide impedance bandwidth is similar to our previously proposed dual-polarized base station antenna [7]. The first resonance (lower frequency) is caused by the radiators themselves, which can be treated as two pairs of dipoles. Therefore, its resonant frequency $(1.8 \mathrm{GHz})$ is determined by the dimension of the loop radiators $\left(\mathrm{L} / 2+\mathrm{L}_{1}+\right.$ $\mathrm{L}_{2}+\mathrm{L}_{3} / 2 \approx 0.261 \lambda_{0}$, where $\lambda_{0}$ is the free-space wavelength at $1.8 \mathrm{GHz}$ ). On the other hand, the second resonance (upper frequency) is introduced by the unexcited radiators. For example, when differential port 1 is excited, the other two loop radiators connected to differential port 2 (unexcited radiators) work as couple elements, which cause the second resonance. However, these two resonant frequencies are too far away from each other. To fix this problem, four extra parasitic elements is 
introduced, which is the main novelty of this work. By adding these parasitic elements, the second resonant frequency can be shifted to a lower frequency, which is greatly influenced by the side length of the loop parasitic elements, $\mathrm{L}_{\mathrm{p}}$. By properly adjusting the dimension of the radiators and the parasitic elements, a good impedance matching across a wide frequency band can be achieved. The principle of the wide impedance bandwidth will be further discussed in Section III. B.

To obtain a stable unidirectional radiation pattern, a stable antenna gain and a stable beamwidth, the proposed antenna is fixed above a rectangular cavity-shaped reflector with dimensions of $140 \mathrm{~mm} \times 140 \mathrm{~mm} \times 6 \mathrm{~mm}\left(1.073 \lambda_{1}\right.$ by $1.073 \lambda_{1}$ by $0.046 \lambda_{1}$, where $\lambda_{1}$ is the free-space wavelength at $2.3 \mathrm{GHz}$ ) instead of a planar one [7] [19], as shown in Figs. 1(a) and (c). The advantage of a cavity-shaped reflector over a planar one has been fully discussed in [7]. The distance between the antenna and the reflector is about $0.261 \lambda_{1}(\mathrm{H}=34 \mathrm{~mm})$. It should be noted that the dimensions of the metal reflector and the distance between the antenna and the reflector in this paper are the same as [7].

\section{PARAMETRIC STUDY}

Our aim is to design a differentially-fed dual-polarized base station antenna to cover $2 \mathrm{G}$ (1710-1920 MHz), 3G (1880-2170 $\mathrm{MHz}$ ) and LTE bands (2300-2400 MHz and 2570-2690 MHz) simultaneously. Therefore, the antenna should have a wide impedance bandwidth (1710-2690 MHz) with $\mathrm{SWR}<1.5$, a great port-to-port isolation $(>25 \mathrm{~dB})$, a high antenna gain $(>8$ $\mathrm{dBi}$ ) and a stable radiation pattern with $3-\mathrm{dB}$ beamwidth of $65^{\circ} \pm 5^{\circ}$ in the horizontal plane over the whole operating frequency band. In order to obtain the best performances of the proposed antenna, a parametric study was carried out by using Ansoft HFSS Solver [20]. In this study, our main purpose is to achieve a wide impedance bandwidth and a stable radiation pattern. According to this goal, some parameters of the loop radiators, the cross-shaped feeding structure, the loop parasitic elements and the rectangular cavity-shaped reflector, etc, were studied. Note that only differential port 1 was studied because port 2 has the same simulated results. Besides, only 3-dB beamwidth in the horizontal plane was investigated since vertical plane has the same results. To understand each parameter's effect on the antenna's performance independently, only one parameter is studied at a time.

\section{A. Loop Radiators and Cross-shaped Feeding Structure}

Fig. 2 shows the effect of the first parameter, $L_{1}$, on antenna gain and differential SWR from 1.5 to $3.1 \mathrm{GHz}$. It can be observed that the antenna has two resonances across the frequency band and the impedance matching is very sensitive to this parameter. When $\mathrm{L}_{1}$ is set to be $8.5 \mathrm{~mm}$, the first resonance occurs at about $1.8 \mathrm{GHz}$ while the second at 2.7 $\mathrm{GHz}$. The curves in Fig. 2 also reveal that as $\mathrm{L}_{1}$ increases both of these two resonances happen at a lower frequency. As mentioned in Section II, the lower resonance is caused by the loop radiators themselves and when their dimensions, determined by $\mathrm{L} / 2+\mathrm{L}_{1}+\mathrm{L}_{2}+\mathrm{L}_{3} / 2$, increases, the resonant

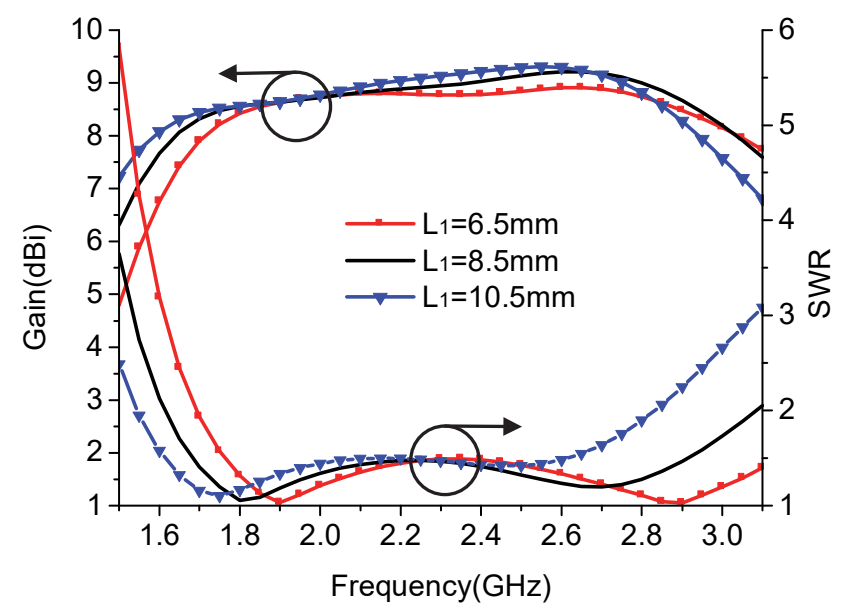

Fig. 2. Antenna Gain and differential SWR versus parameter $L_{1}$.

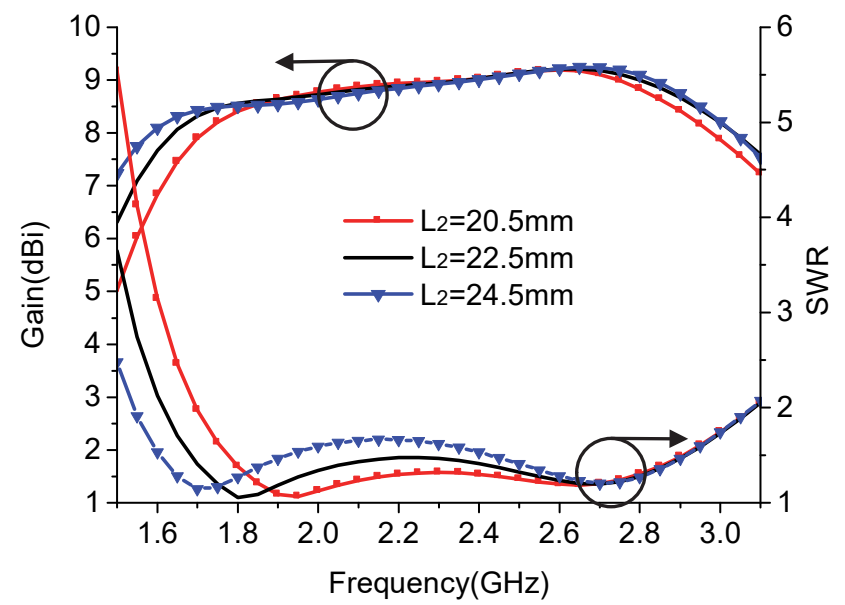

Fig. 3. Antenna Gain and differential SWR versus parameter $\mathrm{L}_{2}$.

frequency obviously should move to a lower frequency. It is analyzed in [7] that the second resonance was caused by the unexcited loop radiators and when the coupling line becomes longer it will shift to a lower frequency, which is demonstrated by Fig. 2. To make the two resonances occur within our designed frequency band, $\mathrm{L}_{1}=8.5 \mathrm{~mm}$ was chosen.

The second parameter that affects the antenna's impedance matching is $\mathrm{L}_{2}$. It can be seen from Fig. 3 that the upper resonant frequency is insensitive to the variation of $\mathrm{L}_{2}$. The reason for this phenomenon is that this resonance is only affected by the coupling between the excited radiators and the unexcited radiators and that between the radiators and the parasitic elements. However, the lower resonance takes place at a lower frequency as the value of $\mathrm{L}_{2}$ is increased. This is because of the increase of the loop radiators' dimensions. Fig. 3 also indicates that the antenna gain is insensitive to $\mathrm{L}_{2}$ across the whole operating frequency band. Thus, $\mathrm{L}_{2}=22.5 \mathrm{~mm}$ was selected to obtain a proper resonant frequency of the lower resonance.

The width of the feeding structure, $\mathrm{W}_{6}$, is another parameter that has a great influence on the antenna's impedance bandwidth. As depicted in Figs. 4, $\mathrm{W}_{6}$ is very important for the impedance matching especially near the upper resonant 


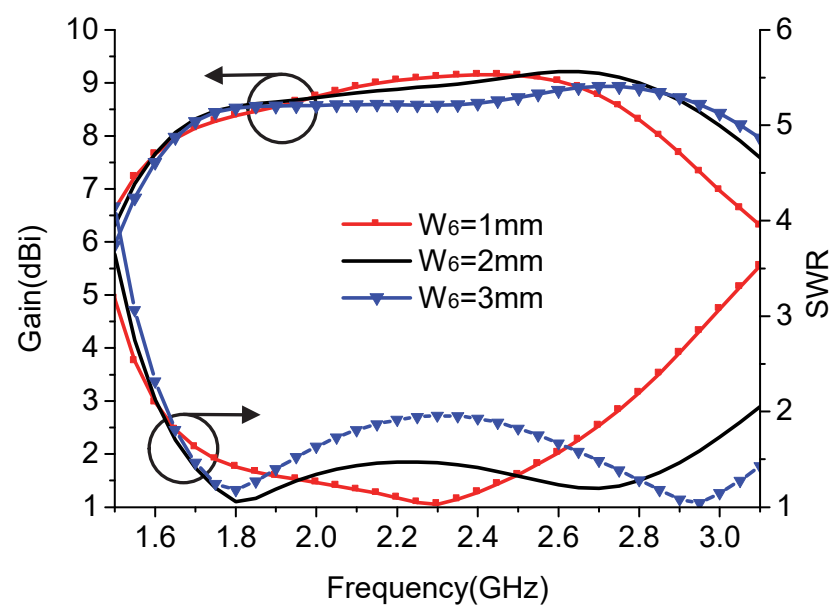

Fig. 4. Antenna Gain and differential SWR versus parameter $\mathrm{W}_{6}$.

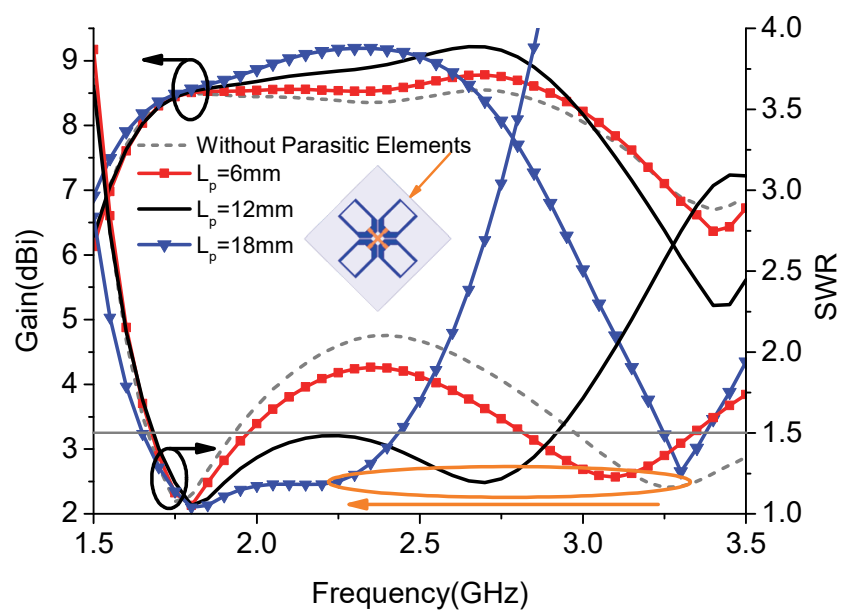

Fig. 5. Antenna Gain and differential SWR versus parameter $L_{p}$.

frequency. As $\mathrm{W}_{6}$ increases, the second resonance occurs at a higher frequency. A low SWR $(<1.5)$ and stable antenna gain (around $8.5 \mathrm{dBi}$ ) can be achieved from 1.7 to $2.9 \mathrm{GHz}$ when $\mathrm{W}_{6}$ $=2 \mathrm{~mm}$ was chosen.

\section{B. Loop Parasitic Elements}

The loop parasitic elements also exert a critical impact on the impedance matching of the antenna especially in the higher frequency band near the second resonant frequency. The effects of varied side length of the loop parasitic element $L_{p}$ on the antenna's SWR and gain are shown in Fig. 5. It can be seen that the impedance matching is very sensitive to this parameter. By increasing the value of $L_{p}$, the upper resonance occurs at a lower frequency while the lower resonance is insensitive to the variation. Fig. 5 also shows that when the proposed antenna does not have four parasitic loop elements, its impedance matching becomes bad with SWR around 2 and at the same time the antenna gain decreases significantly. The reason for this deterioration is that when the parasitic elements are removed, these two resonant frequencies are too far away from each other.

In order to have a distinct view on how the loop parasitic elements affect the proposed antenna's impedance matching, the electrical current distributions of the antenna without

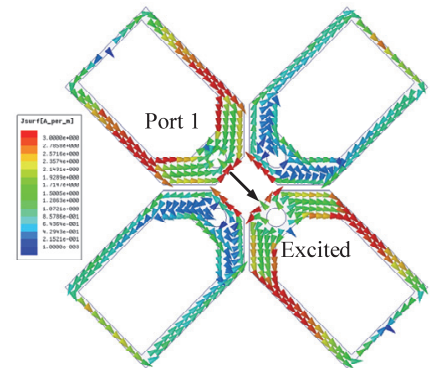

(a)

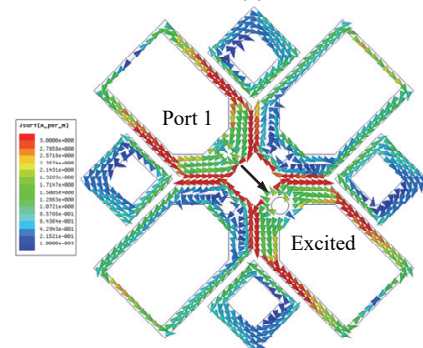

(c)

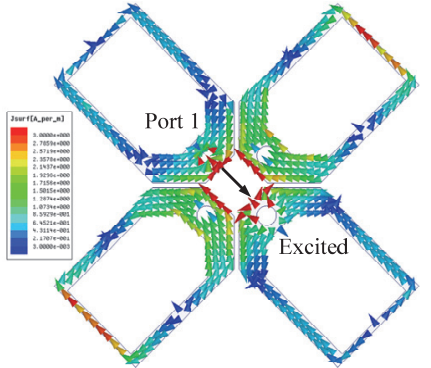

(b)

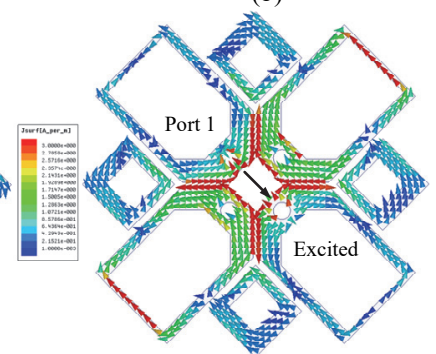

(d)
Fig. 6. Electrical current distributions of antenna without loop parasitic elements at (a) $1.8 \mathrm{GHz}$ and (b) $2.7 \mathrm{GHz}$ and proposed antenna at (c) $1.8 \mathrm{GHz}$ and (d) $2.7 \mathrm{GHz}$.

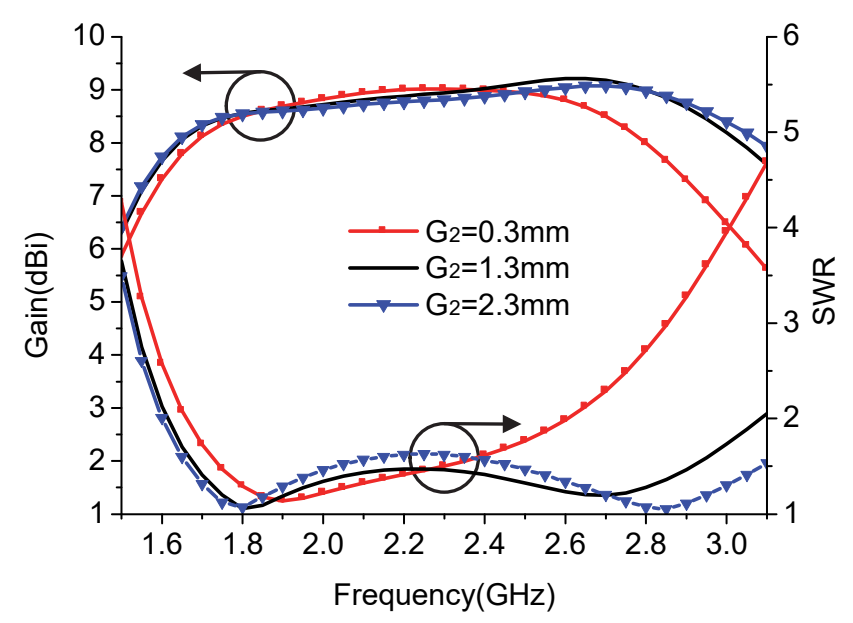

Fig. 7. Antenna Gain and differential SWR versus parameter $\mathrm{G}_{2}$.

parasitic elements and the proposed antenna are compared in Fig. 6. It can be seen from Fig. 6 (a) that for the antenna without parasitic elements, the electrical current at the first resonant frequency of $1.8 \mathrm{GHz}$ is very strong on the excited radiators while the current on the unexcited loops is weak. Therefore, the first resonance was caused by the excited loop dipole itself. When we compare Figs. 6 (a) and (c), it can be observed that the loop parasitic elements almost have no impact on the current distribution at $1.8 \mathrm{GHz}$. That is why they have no impact on the first resonant frequency. However, Figs. 6 (b) and (d) show that the loop parasitic elements have a significant effect on the current distribution at $2.7 \mathrm{GHz}$. Obviously, by adding four parasitic elements, the current on the loop resonators (unexcited loop dipole) is enhanced especially around the feeding points. It means that these parasitic elements can increase the coupling between the excited loop dipole and the unexcited one. As a result, the second resonant frequency 


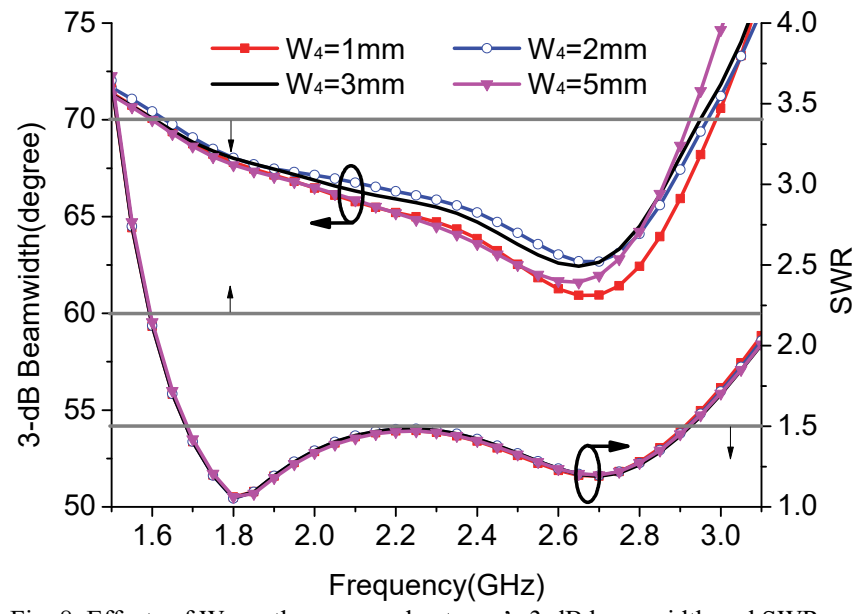

Fig. 8. Effects of $\mathrm{W}_{4}$ on the proposed antenna's 3-dB beamwidth and SWR.

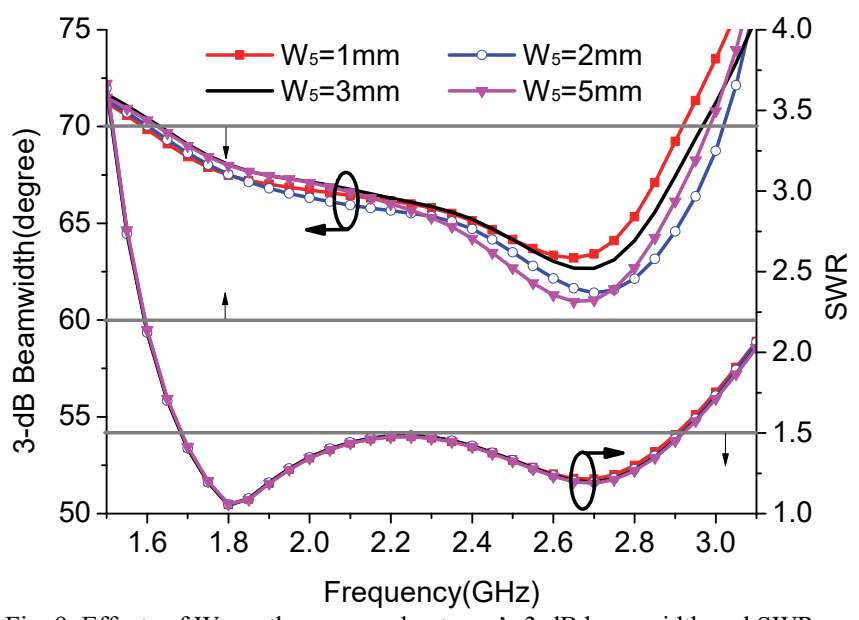

Fig. 9. Effects of $\mathrm{W}_{5}$ on the proposed antenna's 3-dB beamwidth and SWR.

can be shifted down from 3.25 to $2.7 \mathrm{GHz}$ when $\mathrm{L}_{\mathrm{p}}$ was set to be $12 \mathrm{~mm}$ (Fig. 5). Hence, for a wide impedance bandwidth and a high gain for the proposed antenna, $\mathrm{L}_{\mathrm{p}}$ was chosen to be $12 \mathrm{~mm}$.

The second parameter that affects the antenna's impedance bandwidth is the gap between the radiators and the parasitic elements $\mathrm{G}_{2}$. It can be observed in Fig. 7 that a larger $\mathrm{G}_{2}$ yields a wider impedance and gain bandwidth. However, when the gap is too big the impedance matching in the middle of the bandwidth becomes worse. A good impedance matching $(\mathrm{SWR}<1.5)$ and a stable antenna gain (around $8.5 \mathrm{dBi})$ can be achieved from 1.7 to $2.9 \mathrm{GHz}$ when $\mathrm{G}_{2}=2 \mathrm{~mm}$ is selected.

The third and fourth parameters studied are the widths of the loop parasitic elements, $\mathrm{W}_{4}$ and $\mathrm{W}_{5}$. Their effects on the proposed antenna's 3-dB beamwidth and SWR are shown in Fig. 8 and Fig. 9, respectively. It is seen that these two parameters have no impact on the proposed antenna's impedance matching. However, they do have a slight effect on the half power beamwidth of the antenna and $\mathrm{W}_{4}=2 \mathrm{~mm}$ and $\mathrm{W}_{5}=3 \mathrm{~mm}$ are the optimized values.

\section{Rectangular Cavity-Shaped Reflector}

The impact of the rectangular cavity-shaped reflector on the antenna's performances has been fully discussed in [7]. Since the effects of $\mathrm{L}_{\mathrm{g}}, \mathrm{H}_{\mathrm{w}}$, and $\mathrm{H}$ on the proposed antenna's 3-dB beamwidth and antenna gain are similar to [7], they are not repeated here. The optimized values for these three parameters are $140 \mathrm{~mm}, 6 \mathrm{~mm}$ and $34 \mathrm{~mm}$, respectively.

\section{Antenna PERformance}

An antenna prototype was constructed and tested to verify the simulated results. The picture of the antenna prototype is illustrated in Fig. 10. The differential S-parameters are defined as [23],

$$
\begin{aligned}
& S_{d d 11}=\frac{1}{2}\left(S_{11}-S_{12}-S_{21}+S_{22}\right) \\
& S_{d d 22}=\frac{1}{2}\left(S_{33}-S_{34}-S_{43}+S_{44}\right) \\
& S_{d d 21}=\frac{1}{2}\left(S_{31}-S_{41}-S_{32}+S_{42}\right) \\
& S_{d d 12}=\frac{1}{2}\left(S_{13}-S_{14}-S_{23}+S_{24}\right)
\end{aligned}
$$

where $\quad S_{i j}(\mathrm{i}=1,2,3,4 ; \mathrm{j}=1,2,3,4) \quad$ are $\quad$ single-ended S-parameters when the differentially-fed dual-polarized antenna is regarded as a single-ended 4-port network. The single-ended S-parameters were measured by using an Agilent N5230A 2-port vector network analyzer. When two ports were measured, the other two ports were connected with two $50 \Omega$ loads. After obtaining the results of the 4-port S-parameters, the differential S-parameters can be calculated according to expressions (1a) - (1d).

Fig. 11 compares the simulated differential SWRs and port-to-port isolation with the measured results. It can be seen that the simulated and measured SWRs agree well with each other and a wide impedance bandwidth of $52 \%$ with differential $\mathrm{SWR}<1.5$ from 1.7 to $2.9 \mathrm{GHz}$ is achieved at both ports. The measured bandwidth of port 2 becomes wider in the lower frequency, which is caused by fabrication tolerance. The figure also shows that a good port-to-port isolation $(>26.3 \mathrm{~dB})$ is obtained over the operating frequency band for the proposed antenna. Due to the accuracy of the network analyzer and the fabrication tolerance, there is a big difference between the simulated result and the measured one. However, the measured port-to-port isolation is still acceptable. The great port-to-port isolation can be explained by the electrical current distributions. It can be seen that when differential port 1 is excited, the tangential component of the current along the symmetric plane (the solid line) is null. Therefore, the symmetric plane can be treated as a virtual ground and a good isolation between the two differential ports can be observed. It should also be noted that the antenna should have a theoretically infinite differential port-to-port isolation but the simulated result is around $60 \mathrm{~dB}$, which is caused by the finite accuracy of the finite element method (FEM) applied by Ansoft HFSS Solver.

Measurement of the proposed antenna's radiation patterns and antenna gain were accomplished by using a far field antenna measurement system in an anechoic chamber. In order to measure the proposed differentially-fed antenna's far-field 


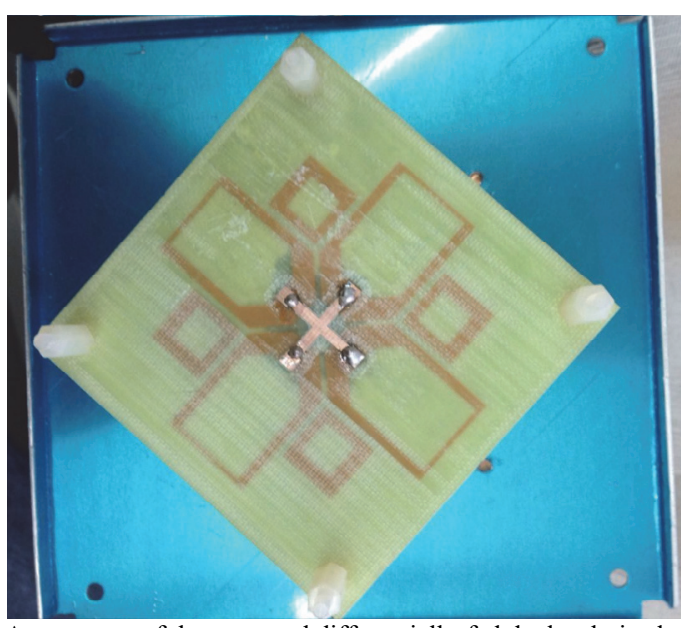

Fig. 10. A prototype of the proposed differentially-fed dual-polarized antenna.

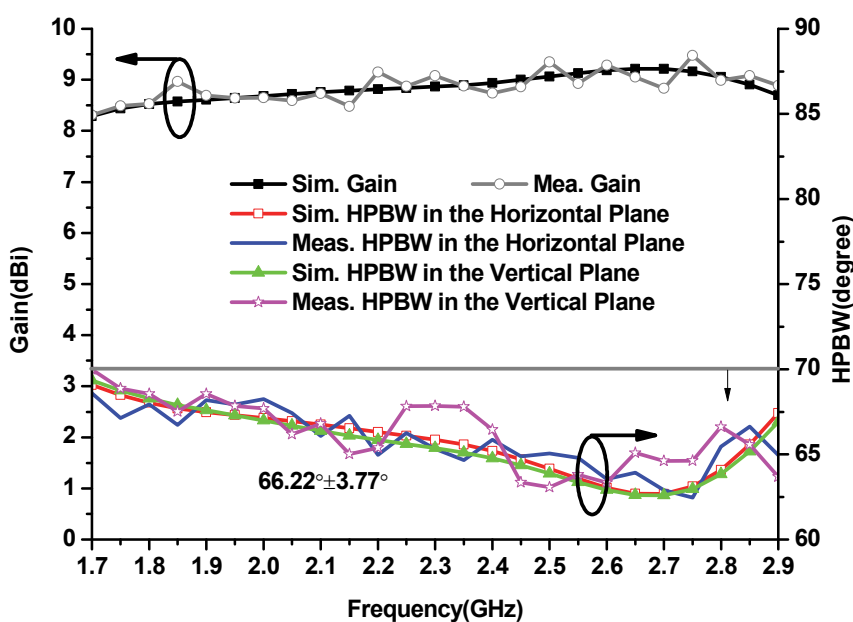

Fig. 12. Simulated and measured antenna gain and $3-\mathrm{dB}$ beamwidths at port 1 .

radiation patterns, a $180^{\circ}$ hybrid coupler was used to transform one single-ended signal to two differential signals. When the radiation patterns of differential port 1 were measured, the two output ports of the $180^{\circ}$ hybrid coupler were connected to port $1^{+}$and port $1^{-}$, respectively, while port $2^{+}$and port $2^{-}$were connected with two $50 \Omega$ loads. It is worthwhile to mention that when the antenna is applied to differential microwave circuits, hybrid coupler is no longer needed.

The simulated and measured antenna gain and 3-dB beamwidths at port 1 and port 2 are shown in Fig. 12 and Fig. 13 , respectively. It can be seen that a good agreement is achieved between simulated and measured antenna gain, which is stable around $8.5 \mathrm{dBi}$ across the entire operating bandwidth. The curves of the simulated and measured half power beamwidths (HPBWs) in Figs. 12 and 13 show a great agreement between the measured and simulated results and both of them are within $65^{\circ} \pm 5^{\circ}$ from 1.7 to $2.9 \mathrm{GHz}$. Figs. 14 and 15 show simulated and measured radiation patterns at port 1 and port 2, respectively. It can be seen that a good agreement is achieved between the simulated and measured results and the co-polarization radiation patterns keep almost unchanged over the operating frequencies. Figs. 12-15 indicate that the antenna has a very stable radiation pattern over its operating frequency

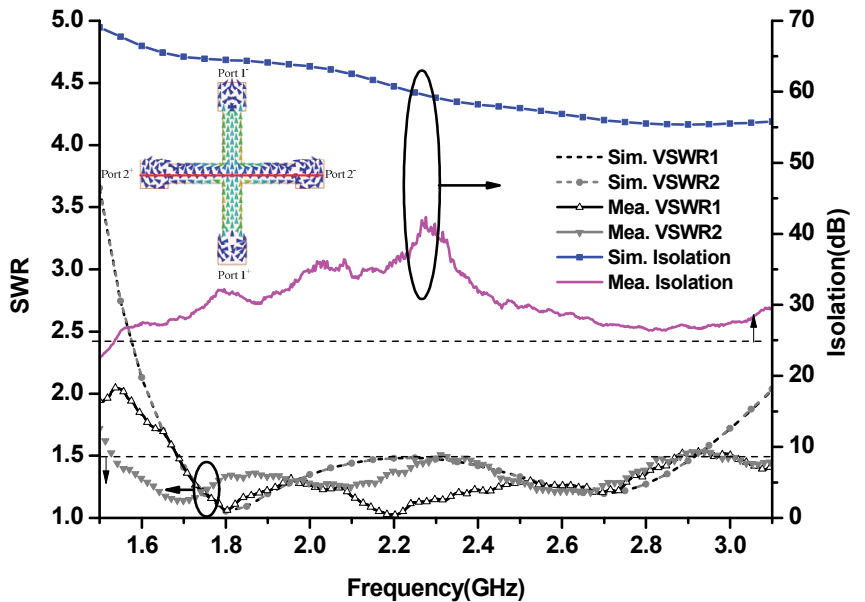

Fig. 11. Simulated and measured SWRs and port-to-port isolation.

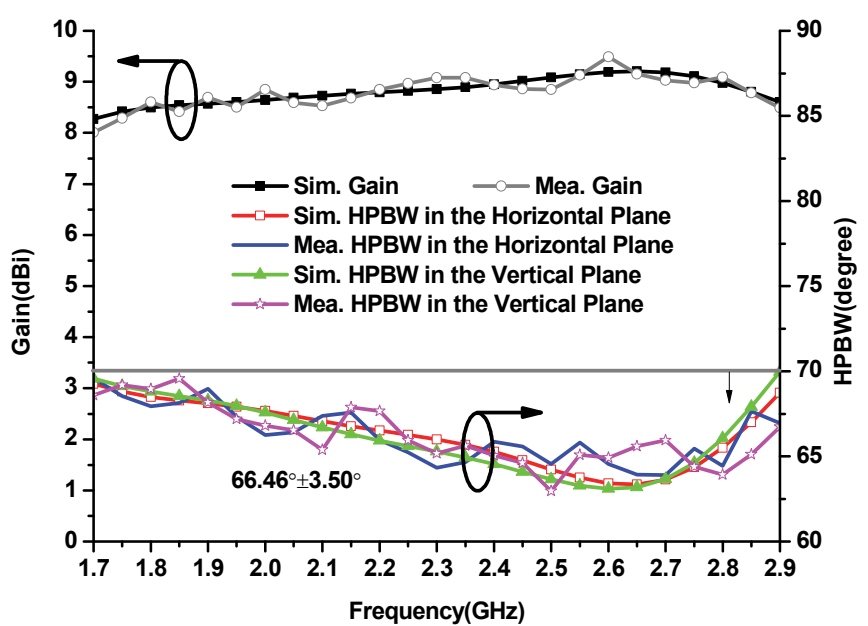

Fig. 13. Simulated and measured antenna gain and $3-\mathrm{dB}$ beamwidths at port 2 .

band.

Table I compares the proposed antenna's performances with other dual-polarized antennas. It can be seen that though the antenna in [7] has the same geometrical dimension with the antenna proposed in this paper, its impedance bandwidth is slightly narrower and it is not differentially driven. When compared with other differentially-fed dual-polarized antennas $[15,16,18]$, the proposed antenna has an advantage of stable radiation pattern and antenna gain over a wide frequency band.

\section{CONCLUSION}

A new broadband differentially-fed dual-polarized antenna with stable radiation pattern has been developed in this paper. After a detailed parametric study to investigate the effects of the loop radiators, the feeding structure, the loop parasitic elements, the rectangular cavity-shaped reflector and the height of the antenna on the antenna's performances, a prototype of it was built and measured. Measurement reveals that the antenna features a wide impedance bandwidth of $52 \%(\mathrm{SWR}<1.5)$ from 1.7 to $2.9 \mathrm{GHz}$, a stable radiation pattern with 3-dB beamwidth of $65^{\circ} \pm 5^{\circ}$, a stable gain (around $8.5 \mathrm{dBi}$ ) and a high port-to-port isolation $(>25 \mathrm{~dB})$ over the operating frequency band. In addition, the proposed antenna can be easily fabricated by 

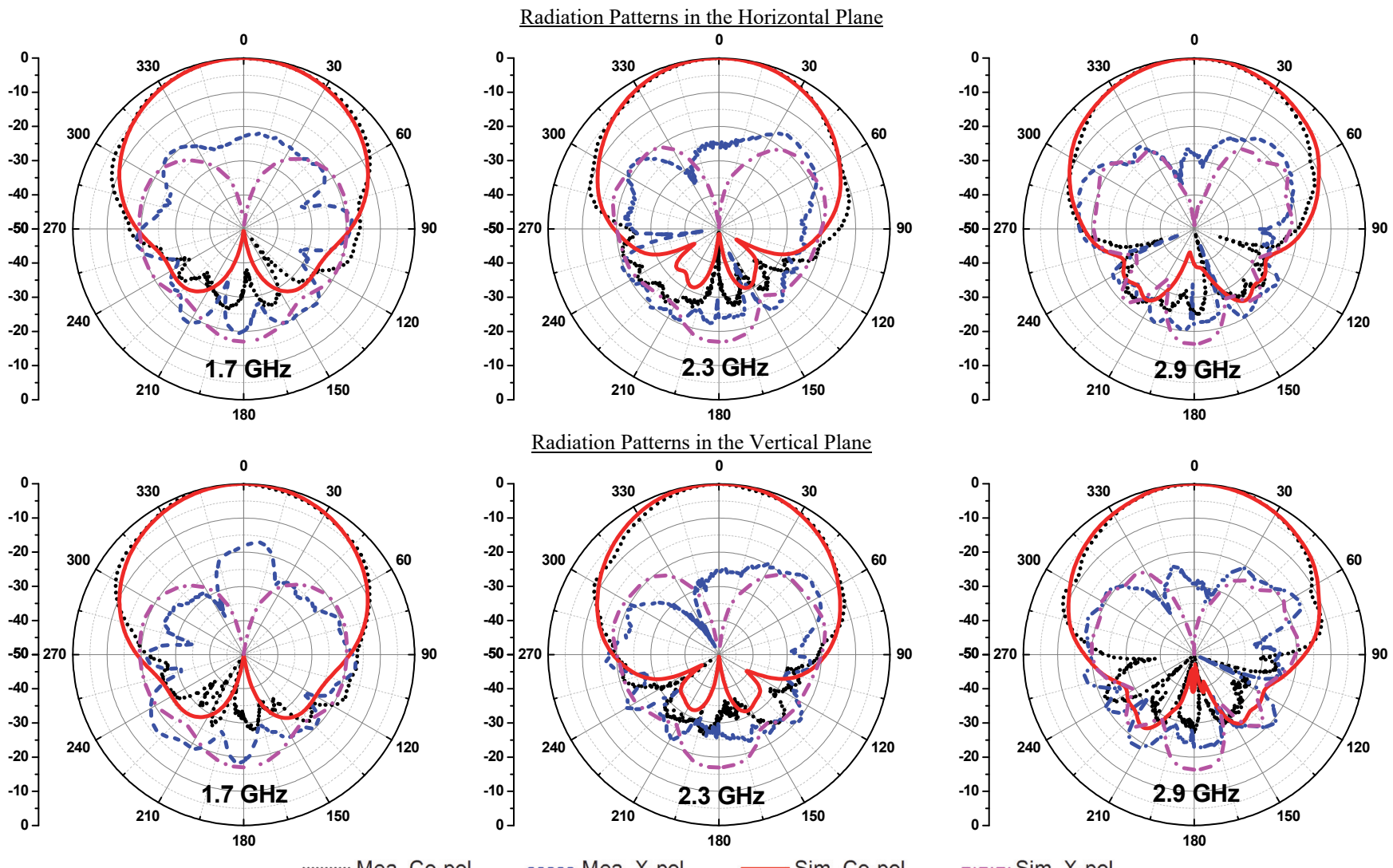

Fig. 14. Simulated and measured radiation patterns at port 1.
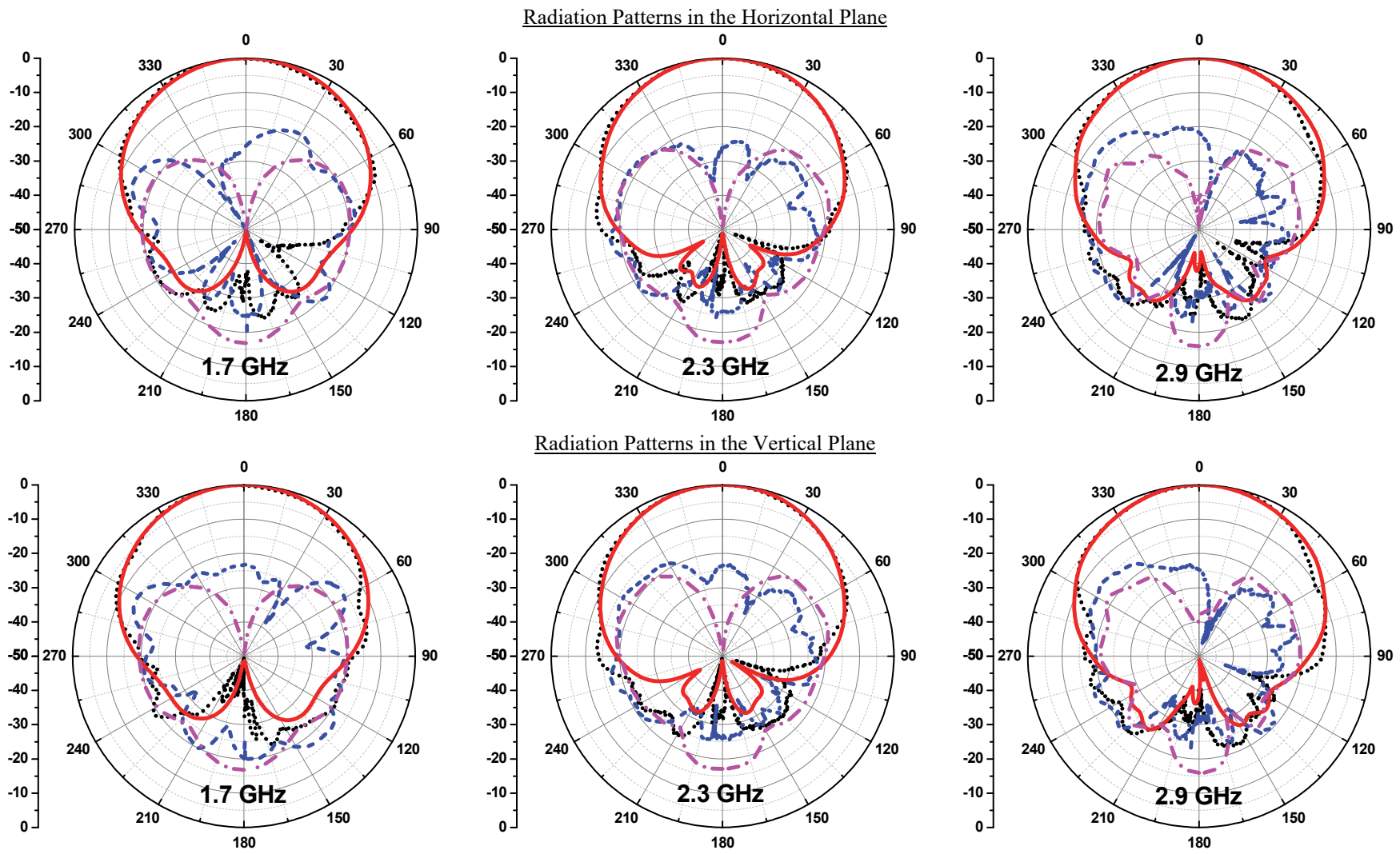

Fig. 15. Simulated and measured radiation patterns at port 2. 
TABLE I

Dual-Polarized AntenNAS' Performance COMPARISON

\begin{tabular}{|c|c|c|c|c|c|c|}
\hline Ref & Differentially-fed & Size $\left(\mathrm{mm}^{3}\right)$ & Bandwidth & Isolation & Horizontal HPBW & Gain \\
\hline$[3]$ & No & $100 \times 100 \times 47$ & $1.71-2.17 \mathrm{GHz}(\mathrm{SWR} \leq 1.4)$ & $>25 \mathrm{~dB}$ & $65^{\circ} \pm 5^{\circ}$ & $\mathrm{NG}$ \\
\hline$[6]$ & No & $200 \times 100 \times 40$ & $1.71-2.39 \mathrm{GHz}(\mathrm{SWR}<1.5)$ & $>25 \mathrm{~dB}$ & $70.0^{\circ} \pm 4.7^{\circ}$ & $>9 \mathrm{dBi}$ \\
\hline$[7]$ & No & $140 \times 140 \times 34.8$ & $1.7-2.7 \mathrm{GHz}(\mathrm{SWR}<1.5)$ & $>25 \mathrm{~dB}$ & $68^{\circ} \pm 2^{\circ}$ & $\sim 8.2 \mathrm{dBi}$ \\
\hline$[10]$ & No & $360 \times 280 \times 45$ & $1.54-2.86 \mathrm{GHz}\left(\left|\mathrm{S}_{11}\right|<-10 \mathrm{~dB}\right)$ & $\mathrm{NG}$ & $95^{\circ} \pm 5^{\circ}$ & $\sim 8 \mathrm{dBi}$ \\
\hline$[15]$ & Yes & $100 \times 100 \times 6.4$ & $\begin{array}{c}\text { Tunable } 0.6 \mathrm{GHz}: 5.4 \mathrm{MHz} ; \\
1 \mathrm{GHz}: 18 \mathrm{MHz}\left(\left|\mathrm{S}_{11}\right|<-10 \mathrm{~dB}\right)\end{array}$ & $>28 \mathrm{~dB}$ & $\mathrm{NG}$ & $\mathrm{NG}$ \\
\hline$[16]$ & Yes & NG & $\mathrm{UWB}$ & $\mathrm{NG}$ & $\mathrm{NG}$ & $\mathrm{NG}$ \\
\hline$[18]$ & Yes & $260 \times 260 \times 48$ & $0.95-1.92 \mathrm{GHz}\left(\left|\mathrm{S}_{11}\right|<-10 \mathrm{~dB}\right)$ & $>36 \mathrm{~dB}$ & $58.5^{\circ} \pm 3.5^{\circ}$ & $6.6-9.6 \mathrm{dBi}$ \\
\hline This Work & Yes & $140 \times 140 \times 34.8$ & $1.7-2.9 \mathrm{GHz}(\mathrm{SWR}<1.5)$ & $>26 \mathrm{~dB}$ & $66.22^{\circ} \pm 3.77^{\circ}$ & $\sim 8.5 \mathrm{dBi}$ \\
\hline
\end{tabular}

using PCB fabrication technique because it has a compact and planar structure. Hence, the antenna should be a good candidate for future base station systems using differential signals.

\section{REFERENCES}

[1] J. N. Lee, K. C. Lee, et al, "Design of the dual-polarized dipole antenna for small base station," Antennas and Propagation (ISAP), 2012 International Symposium on, pp. 1059-1062, 2012.

[2] X. Liu, S. He, H. Zhou, J. Xie, H. Wang, "A novel low-profile, dual-band, dual-polarization broadband array antenna for $2 \mathrm{G} / 3 \mathrm{G}$ base station," in Proc. Inst. Elect. Eng. Int. Conf. on Wireless Mobile \& Multimedia Networks, pp. 1335-1338, Nov. 2006.

[3] D. Su, J. J. Qian, H. Yang and D. Fu, "A novel broadband polarization diversity antenna using a cross-pair of folded dipoles," IEEE Antennas Wireless Propag. Lett., vol. 4, pp. 422-435, 2005.

[4] H. Wang, X. W. Dai and G. Fu, "Design of a novel dual-polarized broad-band base station antenna," Signals Systems and Electronics (ISSSE), 2010 International Symposium on, pp. 1-3, 2010.

[5] Y. H. Huang, Q. Wu and Q. Zh. Liu, "Broadband dual-polarised antenna with high isolation for wireless communication," Electronics Letters, vol. 45, no. 14, pp. 714-715, 2009.

[6] I. Seo, I. Cho, C. Lee, H. Jung, J. Oh, J. Jung, H. Lee, "Design of dual polarized antenna for DCS, UMTS, WiBro base stations," in Proc. IEEE Int. Conf. Wireless Inf. Technol. Syst., pp. 1-4, 2010.

[7] Q. X. Chu, D. L. Wen and Y. Luo, "A Broadband $\pm 45^{\circ}$ Dual-polarized Antenna with Y-shaped Feeding Lines," IEEE Trans. Antennas Propag., vol. 63, no. 2, pp. 483-490, Dec. 2014.

[8] Y. Cui, R. Ling, and H. Fu, "A broadband dual-polarized planar antenna for 2G/3G/LTE base stations," IEEE Trans. Antennas Propag., vol. 62, no. 9, pp. 4836-4840, Sep. 2014.

[9] Z. Bao, Z. Nie, and X. Zong, "A Broadband Dual-Polarization Antenna Element for Wireless Communication Base station," IEEE Asia-Pacific Conference on Antennas and Propagation, pp.144-146, 2012.

[10] Y. Cui, R. Li, and P. Wang, "Novel dual-broadband planar antenna and its array for 2G/3G/LTE base stations," IEEE Trans. Antennas Propag., vol. 61, no. 3, pp. 1132-1139, Mar. 2013.

[11] L. Gao, et al, "Microstrip patch antenna with differential L-Probe Fed For Wideband Application," Cross Strait Quad-Regional Radio Science and Wireless Technology Conference (CSQRWC), 2012. IEEE, 2012.

[12] K. X. Wang, et al, "A novel wide-band patch antenna using differential-fed," CSQRWC, pp. 224-226, 2012.

[13] X. K. Zhang, Z. Chao, et al, "Design of a compact differential dual-frequency antenna," In Cross Strait Quad-Regional Radio Science and Wireless Technology Conference (CSQRWC), 2013, pp. 207-210. 2013.

[14] Z. H. Tu, W. A. Li and Q. X. Chu, "Single-Layer Differential CPW-Fed Notch-Band Tapered-Slot UWB Antenna", IEEE Antennas Wireless Propag. Lett., vol. 13, pp. 1296-1299, 2014.

[15] C. R. White and G. M. Rebeiz, "A differential dual-polarized cavity backed microstrip patch antenna with independent frequency tuning," IEEE Trans. Antennas Propag., vol. 58, no. 11, pp. 3490-3498, Nov. 2010.

[16] G. Adamiuk, W. Wiesbeck, and T. Zwick, "Differential feeding as a concept for the realization of broadband dual-polarized antennas with very high polarization purity," in Proc. IEEE Antennas Propag. Soc. Int. Symp., Charleston, SC, pp. 1-4, Jun. 2009.
[17] M. J. Li and K. M. Luk, "A differential-fed magneto-electric dipole antenna for UWB applications," IEEE Trans. Antennas Propag., vol. 61, no. 1, pp.92-99, 2013.

[18] Q. Xue, S. W. Liao and J. H. Xu, "A differential-driven dual-polarized magneto-electric dipole antenna," IEEE Trans. Antennas Propag., vol. 61 , no. 1, pp. 425-430, 2013.

[19] L. Ge and K. M. Luk, "A Low-Profile Magneto-Electric Dipole Antenna," IEEE Trans. Antennas Propag., vol. 60, no. 4, pp. 1684-1689, Apr. 2012.

[20] AnsoftCorp.HFSS[online].Available:http://www.ansoft.com/products/hf /hfss.

[21] Q. X. Chu, Y. Luo, "A broadband unidirectional multi-dipole antenna with very stable beamwidth," IEEE Transactions on Antennas and Propagation, vol. 61, no. 5, pp. 2847-2852, May. 2013.

[22] C. A. Balanis, Antenna Theory, Analysis and Design, 1997: Wiley.

[23] W. R. Eisenstadt, B. Stengel, and B. M. Thompson, Microwave Differential Circuit Design Using Mixed-Mode S-Parameters. Boston, MA: Artech House, vol. 42-45, pp. 1-25, 2006

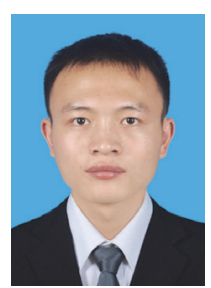

Ding-Liang Wen was born in Ninghua, Fujian Province, China. He received the B.S. degree in electric science and technology and M.E. degree from South China University of Technology, Guangzhou, China, in 2012 and 2015, respectively. Currently, he is working toward the Ph.D degree in electronic engineering at Queen Mary University of London. His research interests include wideband antennas, base station antennas, wearable antennas and theory of characteristic modes.

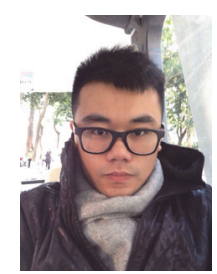

Dong-Ze Zheng was born in Bozhou, Anhui Province, China. He received the B.S. degree in electric science and technology from Anhui University, Anhui, China, in 2014. He is currently working toward the M.E. degree at South China University of Technology, Guangzhou. His research interests include miniature low-profile wideband base station antenna.

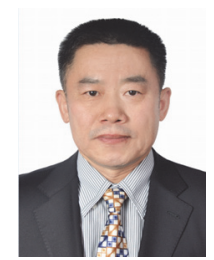

Qing-Xin Chu(M'99-SM'11) received the B.S, M.E., and $\mathrm{Ph} . \mathrm{D}$. degrees in electronic engineering from Xidian University, Xi'an, Shaanxi, China, in 1982, 1987, and 1994, respectively. He is currently a Full Professor with the School of Electronic and Information Engineering, South China University of Technology, Guangzhou, Guangdong, China. He is also the Director of the Research Institute of Antennas and RF Techniques, South China University of Technology. From January 1982 to January 2004, he was with the School of Electronic Engineering, Xidian University. From 1997 to 2004, he was a Professor and later the Vice-Dean with the School of Electronic Engineering, Xidian University. From July 1995 to September 1998 and July to October 2002, he was a Research Associate and Visiting Professor with the Department of Electronic Engineering, Chinese University of Hong Kong, respectively. From February to May 2001 and December 2002 to March 2003, he was a Research Fellow and Visiting Professor with the Department of Electronic Engineering, City University of Hong Kong, respectively. From July to October 2004, he visited the School of Electrical and Electronic Engineering, Nanyang Technological University, Singapore. From January to March 2005, 
he visited the Department of Electrical and Electronic Engineering, Okayama University. From June to July 2008, he was also a Visiting Professor with the Ecole Polytechnique de I'Universite de Nantes, Nantes, France. He has authored o coauthored over 300 papers in journals and conferences. His current research interests include antennas in mobile communication, microwave filters, spatial power-combining array, and numerical techniques in electromagnetics. Prof. Chu is a Senior Member of the China Electronic Institute (CEI). He was the recipient of the Tan Chin Tuan Exchange Fellowship Award, a Japan Society for Promotion of Science (JSPS) Fellowship, the 2002 and 2008 Top-Class Science Award of the Education Ministry of China, and the 2003 First-Class Educational Award of Shaanxi Province. 